\title{
Nuclear and Mitochondrial Suppression of a Mitochondrially Inherited Cold-sensitive Mutation in Aspergillus nidulans
}

\author{
By RICHARD B. WARING* AND CLAUDIO SCAZZOCCHIO \\ Department of Biology, University of Essex, Colchester CO4 $3 S Q$
}

(Received 26 September 1979; revised 14 January 1980)

\begin{abstract}
Partial suppressors of a mitochondrially inherited mutation, [cs-67], conferring coldsensitivity at $20^{\circ} \mathrm{C}$ were identified. These mapped at one mitochondrial and four unlinked nuclear loci. Most suppressors partially restored the cytochrome $a a_{3}$ deficiency of the coldsensitive strain at $20^{\circ} \mathrm{C}$. Strains carrying two or more suppressors and [cs-67] showed considerably impaired growth. This effect was temperature-dependent, being more severe at $37^{\circ} \mathrm{C}$, and was not expressed in the presence of the $\left[c s-67^{+}\right]$allele. The cytochrome oxidase activity of one of these strains was no more heat-sensitive than that of the wild-type implying that these mutations did not directly modify cytochrome oxidase. The wild-type strain grown in the presence of chloramphenicol and the cold-sensitive strain grown at $20^{\circ} \mathrm{C}$ had similar cytochrome spectra and mitochondrial membrane protein profiles on sodium dodecyl sulphate gradient acrylamide gels. [cs-67] conferred pleiotropically a low level of resistance to paromomycin at $37^{\circ} \mathrm{C}$. It is suggested that [cs-67] and the suppressors act at the level of the mitochondrial ribosome.
\end{abstract}

\section{INTRODUCTION}

A cold-sensitive phenotype often results from the inability to assemble a multimeric complex, a classical example being found in mutations affecting the ribosome (for review, see Nomura et al., 1977). In Saccharomyces cerevisiae, a cold-sensitive phenotype can be conferred by a number of mitochondrial mutations, including ones which almost undoubtedly map in the genes specifying the $21 \mathrm{~S}$ mitochondrial ribosomal RNA (BolotinFukuhara et al., 1977; Devenish et al., 1978; Singh et al., 1978) and subunits of the ATPase (Trembath et al., 1975b), cytochrome $b$ and cytochrome oxidase (Bolotin-Fukuhara et al., 1977). It has been established that mitochondrial mutations which confer erythromycin resistance and a cold-sensitive phenotype pleiotropically map in or close to the 21S rRNA gene and generally impair the assembly of the $50 \mathrm{~S}$ mitoribosomal subunit (Singh et al., 1978; Spithill et al., 1978).

In Aspergillus nidulans, Waldron \& Roberts (1973) isolated a cold-sensitive mutant whose phenotype was inherited cytoplasmically. Rowlands \& Turner (1975) showed that the mutation responsible, [cs-67], was on the same linkage group as [oliA1], conferring resistance to oligomycin (Rowlands \& Turner, 1973), and [camA112], conferring resistance to chloramphenicol (Gunatilleke et al., 1975b). The linkage group almost undoubtedly represented the mitochondrial genome (Turner \& Rowlands, 1977, and references therein; Lazarus \& Turner, 1977). Turner \& Rowlands (1976), using low temperature difference spectra, showed that the cold-sensitive strain grown at $20^{\circ} \mathrm{C}$ had an excess of cytochrome $c$, a deficiency in cytochrome $a a_{3}$ and a possible deficiency in cytochrome $b$. They suggested that [cs-67] affected mitochondrial protein synthesis, but from their spectral data could not 
exclude the possibility that [cs-67] specifically impaired the synthesis of cytochrome oxidase. Suppressors of mutations which adversely affect mitochondrial function have been identified in Schizosaccharomyces pombe (Goffeau et al., 1976), Neurospora crassa (Mitchell \& Mitchell, 1956; Kohout \& Bertrand, 1976; Bertrand \& Collins, 1978), Paramecium tetraurelia (Sainsard, 1975) and Saccharomyces cerevisiae (Storm \& Marmur, 1975; Trembath et al., 1975a). The latter two reports described suppressors of thermosensitive mutations.

We proposed to obtain suppressors of [cs-67] with two aims in mind. Firstly, we hoped to detect modifications by the suppressors to specific components of the mitochondrion which were of such a nature that the primary action of [cs-67] could be deduced. Secondly, as the biogenesis of the mitochondrion requires the co-ordinated activity of the nuclear and mitochondrial genomes, transcripts from which appear, in general, to be translated in the cytoplasm and mitochondrion respectively (for review, see Schatz \& Mason, 1974), the identification and characterization of suppressor mutations should facilitate an understanding of the biogenesis of the mitochondrion - specifically, the interaction of the two genetic systems. A similar approach is being pursued in $N$. crassa by Kohout \& Bertrand (1976) who have identified six nuclear suppressors of the mitochondrial mutation poky, which inter alia impairs mitoribosomal assembly.

\section{METHODS}

Strains. As well as markers in general use (Clutterbuck \& Cove, 1974), Aspergillus nidulans strains carried any of the following: oliC1 and [oliA1] - resistance to oligomycin (Rowlands \& Turner, 1973); [camA112] - resistance to chloramphenicol (Gunatilleke et al., 1975b); [cs-67] - cold-sensitivity (Waldron \& Roberts, 1973). Gene symbols representing extranuclear markers are enclosed in square brackets. All strains used for biochemical work and growth rate studies on solid media carried the markers $y A 2$, pyroA4 and cnxC3. In order to facilitate heterokaryon formation, suppressor mutations were placed in both of the following two backgrounds: biA1 luAI cnxH3 and yA2 pyroA4 cnxC3. (biAI, luAI and pyroA4 - nutritional requirements for biotin, leucine and pyridoxine, respectively; $y A 2$ - yellow conidial colour; $c n x C 3$ and $c n x H 3$ - inability to use nitrate or hypoxanthine as nitrogen source.) Strains which were isolated from heterokaryons were purified to ensure that they were homoplasmic.

In vivo techniques. Growth testing and genetic techniques for $A$. nidulans have been described before (Arst \& Cove, 1969, and references therein). Liquid and solid media were from Cove (1966) based on those of Pontecorvo et al. (1953). Urea ( $5 \mathrm{~mm})$ or sodium nitrate $(10 \mathrm{mM})$ were routinely used as the nitrogen source. Carbon sources were added at $1 \%(\mathrm{w} / \mathrm{v})$, except for acetate, which was added as the sodium salt, adjusted to $\mathrm{pH} 6.5$ to give a final concentration of $144 \mathrm{~mm}$. Chloramphenicol and oligomycin resistance were routinely scored on media containing $2.5 \mathrm{mg} \mathrm{ml}^{-1}$ and $3.0 \mu \mathrm{g} \mathrm{ml}^{-1}$, respectively, of the appropriate inhibitor. The marker [cs-67] was scored at $20^{\circ} \mathrm{C}$ on complete medium. Oligomycin, pyrrolnitrin and carboxin were added as stock solutions, dissolved in methanol; as was diuron in acetone. $\mathrm{KCN}$ was always freshly prepared and added as an aqueous solution. Chloramphenicol, salicylhydroxamic acid, neomycin, erythromycin, spiramycin, streptomycin, lincomycin and paromomycin were added as solids to molten agar, cooled to $60{ }^{\circ} \mathrm{C}$. Pyrrolnitrin, diuron, carboxin and paromomycin were gifts from Eli Lilly \& Co. (Indianapolis, Indiana, U.S.A.), du Pont de Nemours \& Co. (Wilmington, Delaware, U.S.A.), Uniroyal Ltd (Bromsgrove, Worcestershire) and Parke, Davis \& Co. (Pontypool, Gwent), respectively. Sodium deoxycholate (Koch-Light) was used at a concentration of $0 \cdot 8 \%(\mathrm{w} / \mathrm{v})$ to induce compact growth on plates (Mackintosh \& Pritchard, 1963).

Growth of mycelium for in vitro studies. Two-litre flasks, each containing $800 \mathrm{ml}$ of complete liquid medium, were inoculated with a conidial suspension to give a final concentration of $2 \times 10^{6} \mathrm{ml}^{-1}$. The flasks were shaken in an orbital shaker at $110 \mathrm{rev} . \mathrm{min}^{-1}$. Growth times were set so that the mycelium was harvested towards the end of the exponential phase, these times being established from appropriate growth curves. Strains carrying the following mutations were grown for the number of hours indicated at 37 and $20^{\circ} \mathrm{C}$, respectively: wild-type $(10 \cdot 5,36 \cdot 0)$; [cs-67] $(10 \cdot 5,64 \cdot 0)$; [cs.67] and one of the suppressors sumA31, sumA35, sumB19, sumB72, sumC40 or [sumD16] $(10 \cdot 5,48 \cdot 0)$; [cs.67] and sumE83 $(10 \cdot 5,56 \cdot 0)$; [cs.67], sumA31 and $\operatorname{sum} B 19(15 \cdot 0,48 \cdot 0) ;[\operatorname{cs}-67]$, sumA35 and $\operatorname{sumB} 19(20 \cdot 0,56 \cdot 0)$. The wild-type grown at $20^{\circ} \mathrm{C}$ in the presence of 2.5 and $6.0 \mathrm{mg}$ chloramphenicol ml-1 was harvested after 48.0 and $64.0 \mathrm{~h}$, respectively.

Heterokaryon formation. Strains carrying complementing nitrate non-utilizing markers and complementing auxotrophic markers were inoculated at exactly the same point on minimal medium containing nitrate as sole nitrogen source (Rever, 1965). Strains carrying biAl luAl cnxH3 and yA2 pyroA4 cnxC3 formed 
balanced heterokaryon cores, giving good cytoplasmic mixing without the need for a transfer step to fresh nitrate medium (Gunatilleke et al., 1975b).

Cytoplasmic inheritance. This was described fully by Gunatilleke et cil. (1975b).

Analysis of mitochondrial recombination using random spore analysis. This was based on the method of Rowlands \& Turner $(1974 b, 1975)$ with some modifications (R. B. Waring \& C. Scazzocchio, unpublished results). In mitochondrial crosses where the phenotype of both strains was similar with respect to the mitochondrial markers (e.g. in heterokaryons between the wild-type and revertants of [cs-67] having a wild-type phenotype), reassortment of the mitochondrial genomes between the nuclear genomes was estimated using an independent marker such as [camA112].

Isolation of mutants. Strains were treated with $N$-methyl- $N^{\prime}$-nitro- $N$-nitrosoguanidine after the method of Alderson \& Hartley (1969). Revertants of [cs-67] were selected on minimal media containing either glucose, ethanol or acetate as carbon source. Mutated conidia were plated at densities of $2 \times 10^{3}$ to $2 \times 10^{4}$ per plate and incubated at $18{ }^{\circ} \mathrm{C}$.

Cytochrome spectra. Room temperature difference spectra were obtained using a Pye Unicam SP1800 spectrophotometer, with a turbid sample attachment. Reduction was achieved using sodium dithionite and oxidation by shaking the sample in air. Crude mitochondrial extracts were prepared as follows. Mycelium ( 1 to $2 \mathrm{~g}$ hand-pressed wet $\mathrm{wt}$ ) was disrupted using an ice-cold pestle and mortar, with sand, in $1.5 \mathrm{vol}$. extraction medium (see below). The slurry was suspended in $30 \mathrm{ml}$ extraction medium, vortexed for $20 \mathrm{~s}$ and then centrifuged twice at $800 \mathrm{~g}$ for $5 \mathrm{~min}$ to remove mycelial fragments. The supernatant was centrifuged at $48000 \mathrm{~g}$ to pellet the mitochondrial fraction; this was resuspended in extraction medium to give 2 to $3 \mathrm{mg}$ protein $\mathrm{ml}^{-1}$ and used at this concentration in the spectral analysis. Ratios of cytochrome $c: a a_{3}$ and $c: b$ were calculated, based on the method of Lambowitz et al. (1972a). These calculations were actually estimates as absorption coefficients for $A$. nidulans were not known. Cytochrome concentrations were measured using the following pairs of wavelengths and absorption coefficients, based on Chance \& Williams (1955): cytochrome $a a_{3}, 608-630 \mathrm{~nm}, \epsilon=161 \mathrm{mmol}^{-1} \mathrm{~cm}^{-1}$; cytochrome $b, 560-575 \mathrm{~nm}, \epsilon=201 \mathrm{mmol}^{-1} \mathrm{~cm}^{-1}$; cytochrome $c, 551-540 \mathrm{~nm}, \epsilon=191 \mathrm{mmol}^{-1} \mathrm{~cm}^{-1}$. As a straight and reproducible baseline was not always obtained, one was artificially derived by joining the reference wavelengths 540,575 and $630 \mathrm{~nm}$, these being virtually isosbestic points.

Thermolability of cytochrome oxidase. A crude mitochondrial extract was made as above, except that the mycelium was disrupted using a glass homogenizer in 10 vol. extraction medium (see below). The mitochondrial extract was layered on to a two-step gradient of 1.20 and $1.60 \mathrm{M}$-sucrose and centrifuged at $48000 \mathrm{~g}$ for $40 \mathrm{~min}$. The brownish mitochondrial band at the $1.20 / 1.60 \mathrm{M}$ interface was removed, and its protein concentration was adjusted to $4 \mathrm{mg} \mathrm{ml}^{-1}$ (determined by Lowry's method). Samples were heated in a water bath for set times and then assayed for cytochrome oxidase activity at $25{ }^{\circ} \mathrm{C}$ using the method of Wharton \& Tzagoloff (1967).

Preparation of mitochondria for gels and enzyme assays. This was based on the methods of Rowlands \& Turner (1974a) and Gunatilleke et al. (1975b). Mycelium (30 to $40 \mathrm{~g}$ hand-pressed wet wt) was washed with deionized water and extraction medium and then suspended in 8 vol. pre-cooled extraction medium [0.44 $\mathrm{M}$ sucrose, $10 \mathrm{~mm}$-Tris, $2 \mathrm{~mm}-\mathrm{Na}_{2}$ EDTA and $0.08 \%(\mathrm{w} / \mathrm{v})$ bovine serum albumin (BSA), fraction V; adjusted to $\mathrm{pH} 7.2$ with $\mathrm{HCl}$ (Lambowitz et al., 1972b; Gunatilleke et al., 1975a)]. All subsequent procedures were carried out at $4{ }^{\circ} \mathrm{C}$. The mycelial suspension was passed twice through a grind-mill (Weiss et al., 1970), shaken in an MSE Atomix blender for $30 \mathrm{~s}$ at half-speed and then filtered through two layers of fine nylon crepe. The filtrate was centrifuged at $800 \mathrm{~g}$ for $10 \mathrm{~min}$ and the resulting supernatant was centrifuged at $21000 \mathrm{~g}$ for $30 \mathrm{~min}$. The reddish-brown pellet obtained was resuspended in extraction medium using a ground glass homogenizer and loaded on to a discontinuous sucrose gradient, which usually consisted of four fractions: $0.90,1.20,1.35$ and $1.60 \mathrm{M}$-sucrose, each containing $10 \mathrm{~mm}$-Tris plus $2 \mathrm{~mm}$-EDTA and adjusted to $\mathrm{pH} 7 \cdot 0$. After centrifugation at $38000 \mathrm{~g}$ for $70 \mathrm{~min}$, the required bands were removed. The mitochondrial fraction banding at the $1 \cdot 20 / 1.35 \mathrm{M}$ interface was the purest on the criterion that it had the highest cytochrome $c$ : cytochrome $b$ ratio.

Preparation of mitochondrial membrane fractions. The purified mitochondrial fractions were washed in extraction medium and centrifuged at $21000 \mathrm{~g}$ for $30 \mathrm{~min}$. A portion of each pellet was resuspended in $10 \mathrm{~mm}$-Tris plus $2 \mathrm{~mm}$-EDTA and sonicated at an amplitude of 8 to $12 \mu \mathrm{m}$ for $15 \mathrm{~s}$. These suspensions were centrifuged at $38000 \mathrm{~g}$ for $30 \mathrm{~min}$ to obtain membrane pellets. The pellets were dissolved in sodium dodecyl sulphate (SDS) sample buffer $(0.05 \mathrm{M}$-Tris/ $\mathrm{HCl} \mathrm{pH} \mathrm{6.8,3 \%} \mathrm{SDS,} 1 \%$ mercaptoethanol, $0.24 \mathrm{M}$-sucrose and $0.05 \%$ bromophenol blue) and heated for $5 \mathrm{~min}$ in a boiling water bath. The final protein concentration was 2 to $4 \mathrm{mg} \mathrm{ml}^{-1}$.

SDS gradient acrylamide gels. The method was based on that of Laemmli (1970). The gradients were 8 to $20 \%$ acrylamide. The gels were approximately calibrated for molecular weight using a preparation of beef heart cytochrome oxidase [a gift of W. Lalla-Maharajh of the Department of Chemistry, University of 
Essex, prepared using a modified method of Yonetani (1960)] and the following standards: bovine serum albumin, fraction V (mol. wt 68000), ovalbumin (43000), aldolase (40000), lactate dehydrogenase (36000), carbonic anhydrase (29000), chymotrypsinogen (25700), myoglobin (17200), ribonuclease A (13700) and cytochrome $c(11700)$. Cytochrome $c$ was obtained from Calbiochem and the other standards were from Sigma. It was considered that cytochrome $c$ and ribonuclease A ran anomalously on the basis of the position of beef heart cytochrome oxidase subunits, whose molecular weights have been well characterized (Briggs \& Capaldi, 1977), and the fact that myoglobin (mol. wt 17200) ran to the same position as ribonuclease A (mol. wt 13700), noting that cytochrome $c$ and ribonuclease A, but not myoglobin, have previously been reported to run anomalously (Dunker \& Rueckert, 1969; Lambin et al., 197ó).

Enzyme assays and protein determination. Cytochrome oxidase was assayed according to the method of Ferguson-Miller et al. (1976), employing an oxygen electrode. The reaction was started by adding the mitochondrial preparation. After $50 \%$ of the oxygen had been consumed, $\mathrm{KCN}$ at a final concentration of $1 \mathrm{mM}$ was added. The rate of cyanide-sensitive oxygen utilization was measured. Succinate dehydrogenase was assayed as described by Butcher (1970) and succinate cytochrome $c$ reductase as described by King (1967). Protein concentrations were determined by the method of Lowry.

\section{RESULTS}

Revertants of [cs-67] were isolated at $18{ }^{\circ} \mathrm{C}$ from mutagenized strains carrying the markers biA1, luA1, cnxH3 and [cs-67]. Each of the 43 isolated was tested for cytoplasmic inheritance of the revertant phenotype by being set in a heterokaryon with the strain $y A 2$ pyroA4 cnXC3 [camA112 cs-67] (see Methods). In 22 cases the revertant and cold-sensitive phenotype segregated in both of the two nuclear backgrounds, demonstrating that the revertant phenotype was cytoplasmically inherited. The remaining 21 revertants were sexually crossed to the strain $y A 2$ pyroA4 cnxC3 [camA112 cs-67]. Progeny with cold-sensitive and revertant phenotypes segregated in a $1: 1$ ratio in each cross, demonstrating that each of these revertants carried a nuclear suppressor.

\section{Preliminary phenotypic and genotypic analysis of nuclear suppressors}

None of the nuclear suppressors fully suppressed [cs-67] at $20^{\circ} \mathrm{C}$. The suppressors mapped at four unlinked loci, which were designated $\operatorname{sum} A, \operatorname{sum} B, \operatorname{sum} C$ and $\operatorname{sum} E$. sum $A$ had 16 alleles. Growth of strains carrying [cs-67] and sumA35 or sumA48 was slightly poorer than that of the wild-type at $37^{\circ} \mathrm{C}$ on complete medium but not synthetic complete medium. The impaired growth phenotype was not separable from the suppressor phenotype in 100 and 36 progeny, respectively. These two mutants were indistinguishable from the other sum $A$ mutants at $20^{\circ} \mathrm{C}$. sum $A 35$ and sum $A 48$ were not separable by recombination from sumA23 in 1000 progeny tested, nor were any of the other sum $A$ mutations in 50 to 800 progeny tested. $\operatorname{sum} B$ had two alleles, $\operatorname{sum} B 19$ being a slightly better suppressor than sumB $B 2$. These two mutations were not separable by recombination in 351 progeny tested. The sumC and sumE loci had one representative each. sumE83 was considerably less effective as a suppressor than the other mutations. The phenotypes of the suppressors are shown in Fig. 1.

Haploidization analysis (McCully \& Forbes, 1965) located sumA on linkage group VII, $\operatorname{sum} B$ on group III, sum C on group IV and sumE on group I. None of the nuclear suppressors have been meiotically mapped as yet, although linkage of sumA35 to the unmapped mutation $c b x B 28$, which confers resistance to carboxin (Gunatilleke et al., 1975a), was observed with a recombination frequency of $11 \%$.

sumA35 recombined freely with oliC1, a mutation conferring oligomycin resistance located in linkage group VII (Rowlands \& Turner, 1973), while sumB19 recombined freely with plnA18 and $c b x A 17$, also on linkage group III and conferring, respectively, pyrrolnitrin and carboxin resistance (Gunatilleke, 1974; Gunatilleke et al., 1975a).

The nuclear suppressors were tested for dominance by constructing homozygous and heterozygous suppressor diploids in a [cs-67] background and comparing growth of the heterozygote with respect to that of the homozygote and a cold-sensitive diploid. In a 


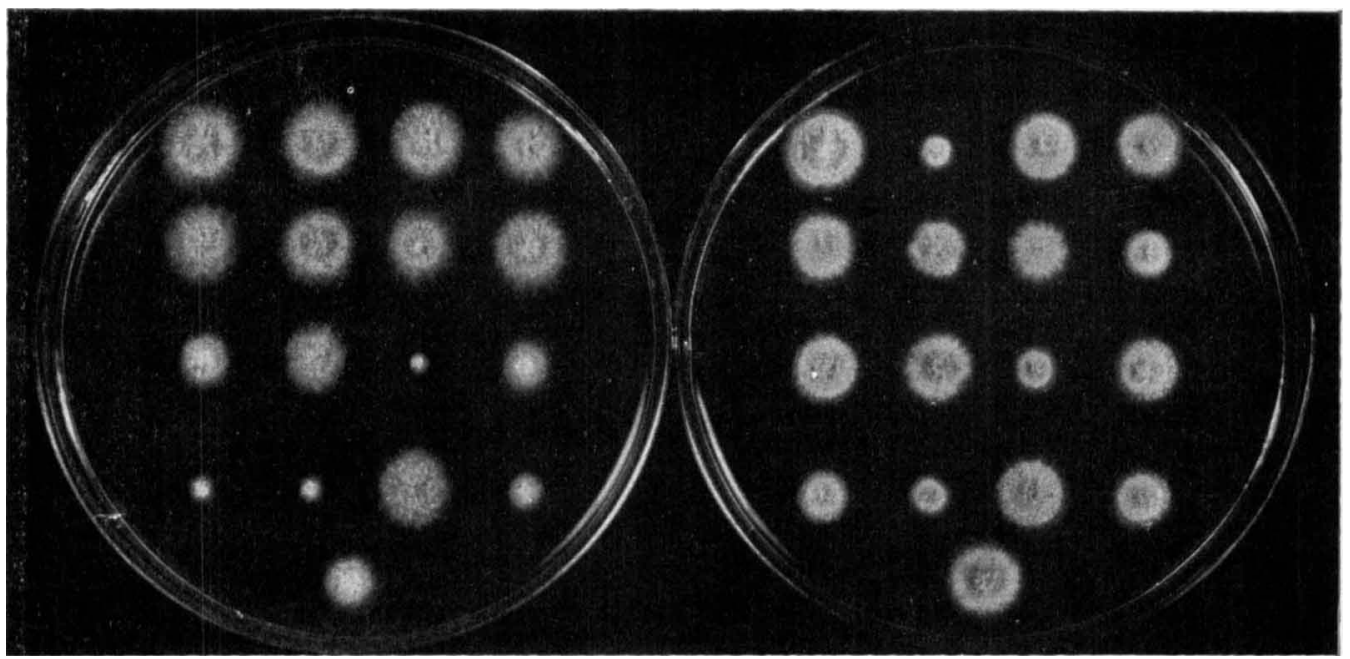

Fig. 1. Growth of suppressor mutants on complete medium at $37^{\circ} \mathrm{C}(l \mathrm{l} f t)$ and $20^{\circ} \mathrm{C}($ right $)$. Relevant genotypes are given below in the same pattern as on the plates.

$\begin{array}{lllll}\text { 1st row: } & \text { wild-type } & {[c s-67]} & \text { sum A31 [cs-67] } & \text { sumA35 [cs-67] } \\ \text { 2nd row: } & \text { sumB19[cs-67] } & \text { sumB72 [cs-67] } & \text { sumC40[cs-67] } & \text { sumE83 [cs-67] }\end{array}$

3rd row: sumA31 sumB19 [cs-67] sumA31 sumE83 [cs-67] sumA35 sumB19 [cs-67] sumA35 sumB72 [cs-67] 4th row: sumA35 sumC40 [cs-67] sumA35 sumE83 [cs-67] sumB19 sumC40 [cs-67] sumB19 sumE83 [cs-67] 5th row: $\quad$ sumC40 sumE83 [cs-67]

[cs-67] background, at $20^{\circ} \mathrm{C}$, heterozygous diploids of sumA23, sumA31, sumA35, sumB19, sumB72, sumC40 and sumE83 all showed intermediate growth between that of their respective homozygous diploid (sum/sum) and the cold-sensitive diploid $\left(\mathrm{sum}^{+} / \mathrm{sum}^{+}\right)$. The suppressor alleles mapping at the same locus were tested in heteroallelic diploids in a [cs-67] background for positive and negative complementation, but no indication of either of these was found.

From heterokaryons between wild-type strains and mutants carrying a suppressor mutation and [cs-67], strains carrying a suppressor and $\left[c s-67^{+}\right]$were isolated. These were all phenotypically indistinguishable from the wild-type strain at 20 and $37^{\circ} \mathrm{C}$.

\section{Genetic analysis of revertants with extranuclear mode of inheritance}

Each revertant was set in a heterokaryon with a strain having the mitochondrial genome [camA112]. Using the random spore analysis technique (Rowlands \& Turner, 1974b, 1975; R. B. Waring \& C. Scazzocchio, unpublished results), the conidia of each heterokaryon were screened for the cold-sensitive phenotype, as this would indicate that the [cs-67] marker had segregated from a suppressor mutation. In the mitochondrial crosses involving 19 revertants, no cold-sensitive recombinants were observed in 100 to 1500 reassorted colonies. These mutants therefore carry either back-mutations or suppressor mutations which are extremely tightly linked to [cs-67], the former explanation being more probable as the phenotype of each mutant was indistinguishable from the wild-type at 37 and $20^{\circ} \mathrm{C}$. Between 3 and $10 \%$ of reassorted colonies from heterokaryons involving revertants 12,16 and 74 were cold-sensitive recombinants, inferring that the revertants carried suppressor mutations. This conclusion was consistent with the observation that these three mutations only partially suppressed [cs-67]. As the suppressor mutations were not easily separable by recombination from [cs-67], they almost undoubtedly map in the mitochondrial genome.

To see if the suppressor mutations themselves were separable by recombination, mitochondrial crosses were conducted using a marker selection technique, the use of which 
facilitates the detection of rare recombinational events. Heterokaryons were set in order to carry out mitochondrial crosses such as [camA112 cs-67 sum-16] to [oliA1 cs-67 sum-74]. Conidia were plated on media containing chloramphenicol and oligomycin in order to select for [camA112 oliA1] recombinants (the growth temperature was non-restrictive). These were then screened for the occurrence of a recombinational event between [sum-74] and [sum-16] by looking for cold-sensitive colonies. The advantage of this method can be seen if one considers the frequency with which recombination between two unlinked markers will be observed. As at least one mitochondrial recombinational event will have occurred in every [camA112 oliA1] recombinant, two unlinked markers will be likely to show recombination in $50 \%$ of the colonies analysed. By comparison, in random spore analysis recombination between two unlinked markers will only be observed in about $5 \%$ of all colonies analysed (Rowlands \& Turner, 1975).

In mitochondrial crosses of [oliA1 cs-67 sum-16] to [camA112 cs-67 sum-12] and [camA112 cs-67 sum-74], no cold-sensitive colonies were observed in $545(<0.2 \%)$ and $353(<0.3 \%)$ [camA112 oliA1] recombinants, respectively. As a control, the linkage between [cs-67] and [sum-16] was also estimated using double marker selection analysis. In a mitochondrial cross such as [oliA1 cs-67 sum-16] to [camA112], recombination between [cs-67] and [sum-16] took place in 8 to $15 \%$ of [camA112 oliAl] recombinants. As these two markers are loosely linked (R. B. Waring \& C. Scazzocchio, unpublished results), the three mitochondrial suppressors almost undoubtedly map in the same gene. This was named [sumD]. As the above results strongly suggest that these three mutations are allelic and as they confer the same phenotype at 37 and $20^{\circ} \mathrm{C}$, only [sumD16] was used for further studies.

In the mitochondrial cross $[c s-67 \operatorname{sum} D 16]$ to $\left[c s-67^{+} \operatorname{sum} D^{+}\right]$, no novel phenotype corresponding to the [sumD16] genotype was observed and so it was initially assumed that such a strain would have a wild-type phenotype. Strains carrying [sumD16] were isolated from four-point mitochondrial crosses (R. B. Waring \& C. Scazzocchio, unpublished results). Putative [sumD16] strains were checked by establishing heterokaryons with strains carrying [cs-67] and scoring for the presence of strains showing the suppressed phenotype. Two of the putative [sumD16] strains yielded such suppressed colonies, establishing that [sumD16] single mutants have the wild-type phenotype.

\section{Interaction of suppressor mutations with other mutations affecting mitochondrial function}

In a [cs-67] background, the suppressors sumA35, sumB19 and [sumD16] did not interact with the following mutations, which are supposed to affect mitochondrial function: $p \ln A 18$, $c b x A 17, c b x B 28$ and oliC2, conferring pyrrolnitrin, carboxin and oligomycin resistance, respectively (the action of the inhibitors is described below). The effect of the suppressors upon the mitochondrial mutations [camA112] and [oliA1] was tested by transferring these two markers into each single suppressor strain carrying [cs-67] and one of the suppressors sumA31, sumA35, sumB19, sumB72, sumC40, sumE83 or [sumD16]. Strains were tested on media with and without chloramphenicol and oligomycin at 37 and $20^{\circ} \mathrm{C}$. No significant effects were seen. At $20^{\circ} \mathrm{C}$ [ camA112] slightly reduced the growth rate of all strains carrying [cs-67], including the cold-sensitive strain itself.

\section{Phenotypes of strains containing more than one suppressor mutation}

When strains containing suppressor mutations at more than one chromosomal locus were constructed in a [cs-67] background, a severe reduction in the rate of growth at $37^{\circ} \mathrm{C}$ occurred, this result being more striking on complete medium than on synthetic complete medium. The effect on growth rate of the combinations of different suppressors is shown in Table 1 and selected examples are shown in Fig. 1. It must be stressed that this reduction in the rate of growth was strictly dependent on the presence of [cs-67] and was absent in a $\left[c s-67^{+}\right]$background. Moreover, for $\operatorname{sum} A$ and $\operatorname{sumB}$, where more than one allele was available, it was clear that growth impairment was allele-specific, sumA35 and sumB19 


\section{Table 1. Impairment of growth in mutants carrying [cs-67] and two nuclear suppressor mutations}

Every double suppressor mutant carried the [cs-67] mutation. Each mutant was scored on the scale 1 to 10 for its growth on solid complete medium at $37^{\circ} \mathrm{C}$. The wild-type and [cs-67] strain were both scored as 10 .

\begin{tabular}{lcccccc} 
Suppressor & \multicolumn{7}{c}{ Growth score } & & \\
mutation & sumA35 & sumB19 & sumE83 & sumA31 & sumC40 & sumB72 \\
sumA35 & & 1 & 2 & - & 2 & 6 \\
sumB19 & 1 & & 3 & 6 & 10 & - \\
sumE83 & 2 & 3 & & 8 & 7 & 10 \\
sumA31 & - & 6 & 8 & & 9 & 10 \\
sumC40 & 2 & 10 & 7 & 9 & & 10 \\
sumB72 & 6 & - & 10 & 10 & 10 &
\end{tabular}

reducing growth more drastically than $\operatorname{sum} A 31$ and $\operatorname{sumB} 72$, respectively. The results from Table 1 established the following general order in the degree of impairment in growth: $\operatorname{sum} A 35>\operatorname{sum} B 19>\operatorname{sumE} 83>\operatorname{sum} A 31>\operatorname{sum} C 40>\operatorname{sum} B 72$. All strains in Table 1 that appeared as exceptions to this order carried sumC40.

Strains containing one suppressor and the mitochondrially inherited [sumD16] were also impaired in growth at $37^{\circ} \mathrm{C}$ when [cs-67] was present, the one exception being sumB72 [cs-67 sumD 16], where the impairment was marginal. [sumD16] further reduced the rate of growth of sumA31 sumB19 [cs-67], sumA35 sumB19 [cs-67] and sumA35 sumC40 [cs-67] (other combinations were not tested). Even this very drastic reduction in growth was dependent on the presence of the [cs-67] allele.

For all combinations of nuclear suppressors, the impairment of growth at $20{ }^{\circ} \mathrm{C}$ was consistent with, but less obvious than, that at $37^{\circ} \mathrm{C}$ because the effects of suppression and impairment of growth acted in opposite directions. Impairment of growth at $20^{\circ} \mathrm{C}$ was scarcely noticeable in strains carrying a nuclear suppressor together with [cs-67] and [sumD16].

\section{In vivo characterization of suppressor mutations of [cs-67]}

These studies were performed on solid media to test the behaviour of the suppressor mutants under a variety of conditions. The strains tested included those shown in Fig. 1 and strains carrying a single suppressor, having the mitochondrial genome of, firstly, $\left[c s-67^{+}\right]$and, secondly, [cs-67 sumD16]. Strains were tested at 37 and $20^{\circ} \mathrm{C}$.

Carbon source. The effects of glucose, ethanol, acetate and galactose were compared. No significant differences were observed.

Temperature. The strains were grown at $44,37,25,20$ and $15^{\circ} \mathrm{C}$. All strains could still grow at $15^{\circ} \mathrm{C}$. The growth test at $44^{\circ} \mathrm{C}$ revealed that strains carrying [cs-67] and one of the suppressors sumA35, sumB19 or sumC40 were slightly heat-sensitive.

Inhibitors of respiration and oxidative phosphorylation. The following inhibitors were tested : oligomycin - an inhibitor of ATPase (Lardy et al., 1958); carboxin - an inhibitor of succinate dehydrogenase (Gunatilleke et al., 1975a); pyrrolnitrin - a multiple site inhibitor between ubiquinone and cytochrome $b$ (Warden \& Edwards, 1976); diuron - an inhibitor of complex III (Inoue et al., 1967); salicylhydroxamic acid - an inhibitor of the alternative oxidase pathway (Henry \& Nyns, 1975); KCN - an inhibitor of cytochrome oxidase. The inhibitors were used at a variety of concentrations in synthetic complete and complete media but none of the strains was either resistant or hypersensitive at either 37 or $20^{\circ} \mathrm{C}$.

Inhibitors of protein synthesis. Many inhibitors of protein synthesis in E. coli are known to affect yeast mitochondrial ribosomes (Grivell et al., 1973). In E. coli, some of these, at low concentration, can suppress the phenotypes conferred by mutations in protein synthesis (Kirschmann \& Davis, 1969; Gorini, 1974). Suppressor strains were screened for hypersensitivity and resistance to a number of inhibitors, looking especially for phenotypic 

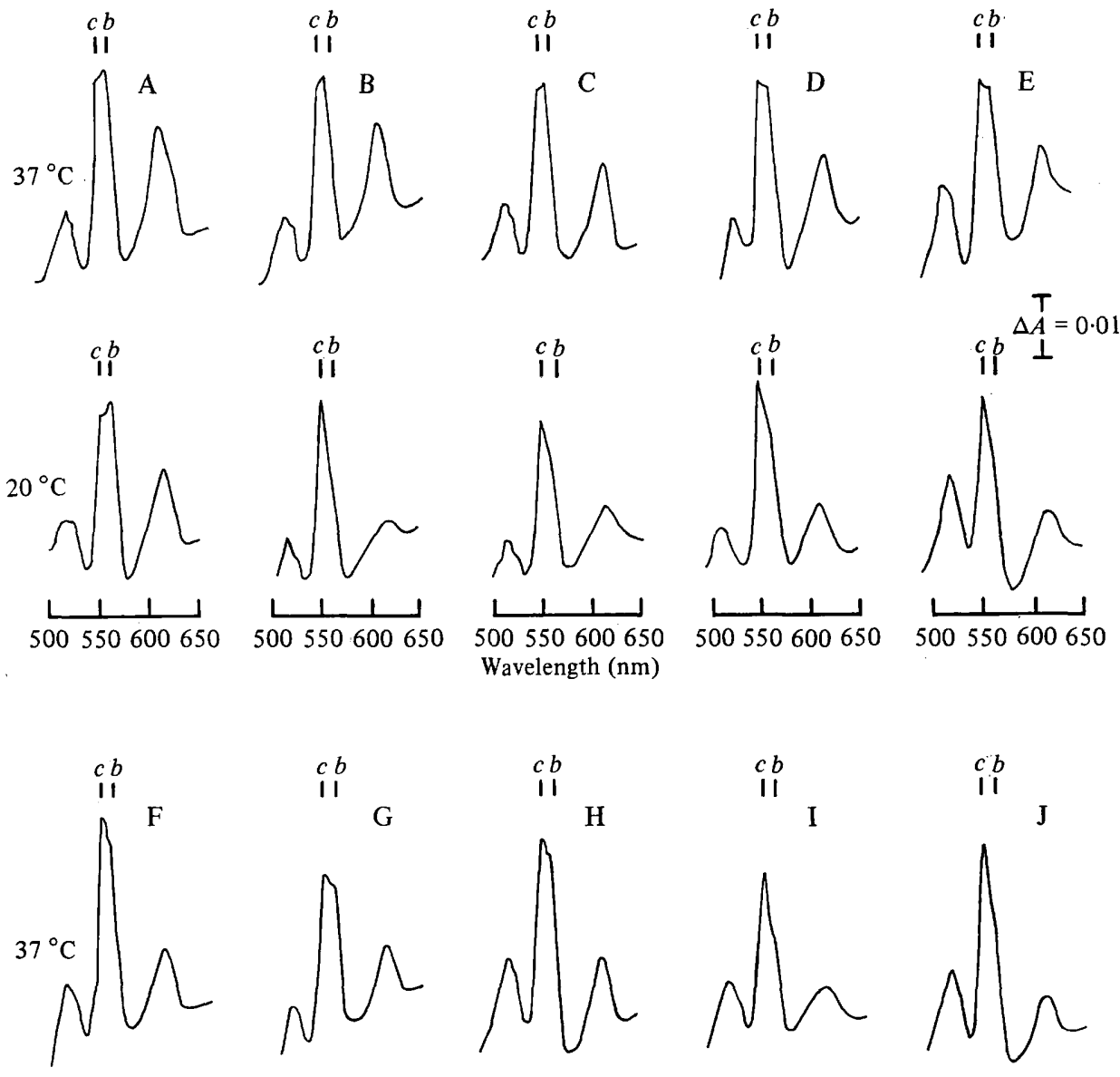

$c b$
11

G
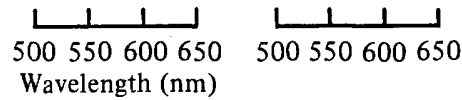

$\underset{500550600650}{L}$

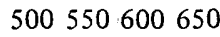

Wavelength (nm)
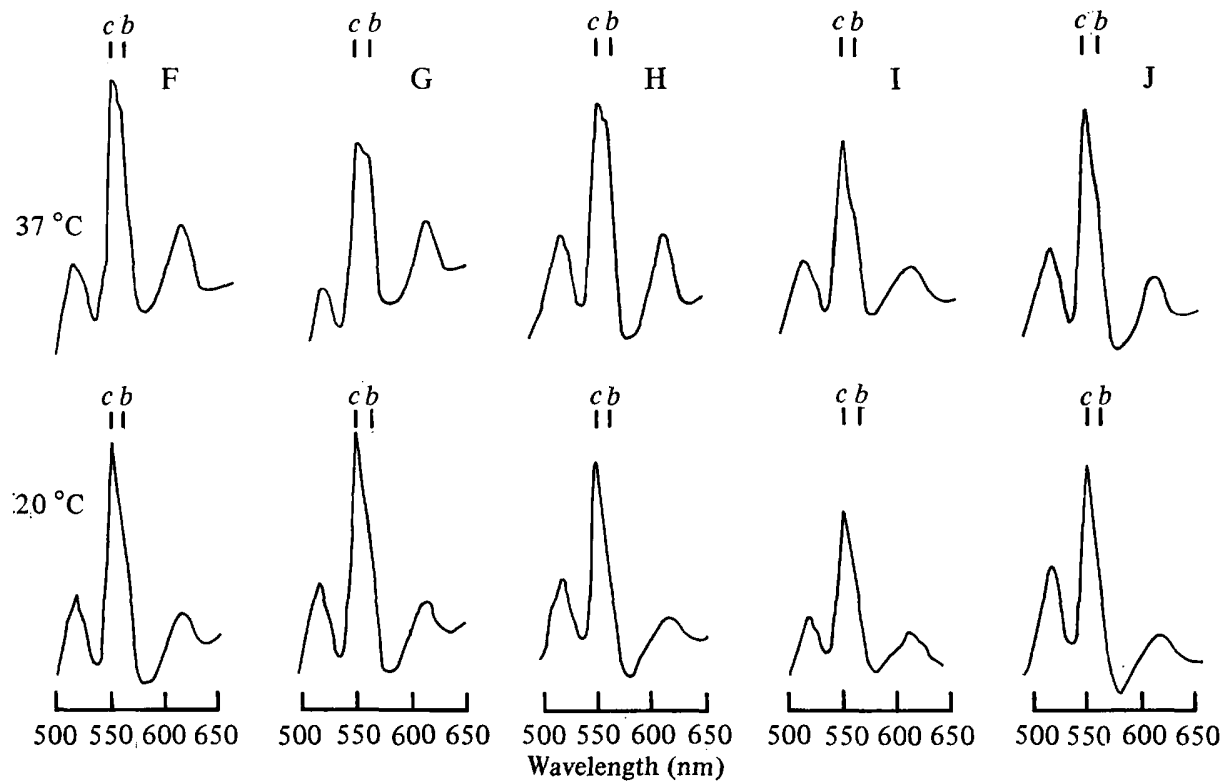

Fig. 2. Room temperature difference spectra of suppressor mutants grown at 37 and $20^{\circ} \mathrm{C}$ : A, wild-type; B, [cs-67]; C, [cs-67 sumD16]; D, sumA31 [cs-67]; E, sumA35 [cs-67]; F, sumB19 [cs-67]; G, sumC40 [cs-67]; H, sumE83 [cs-67]; I, sumA31 sumB19 [cs-67]; J, sumA35 sumB19 [cs-67].

suppression of impaired growth. Tests were carried out on complete and synthetic complete media at 20 and $37^{\circ} \mathrm{C}$. Inhibitors and the concentrations used were: chloramphenicol $\left(1.2,2.5\right.$ and $\left.4.0 \mathrm{mg} \mathrm{ml}^{-1}\right)$, neomycin $\left(0.5,0.8,1.0\right.$ and $\left.2.0 \mathrm{mg} \mathrm{ml}^{-1}\right)$, paromomycin $(0.2$ to 1.0 at 0.1 intervals, 2.0 and $\left.5.0 \mathrm{mg} \mathrm{ml}^{-1}\right)$, erythromycin $\left(5.0\right.$ and $\left.10.0 \mathrm{mg} \mathrm{ml}^{-1}\right)$, lincomycin $\left(5.0 \mathrm{mg} \mathrm{ml}^{-1}\right)$, streptomycin $\left(5.0\right.$ and $\left.10.0 \mathrm{mg} \mathrm{ml}^{-1}\right)$ and spiramycin $\left(5.0\right.$ and $\left.10.0 \mathrm{mg} \mathrm{ml}^{-1}\right)$. Only the first three drugs strongly inhibited the wild-type strain.

[cs-67] conferred a low level of resistance to paromomycin at $37^{\circ} \mathrm{C}$, the cold-sensitive phenotype co-segregating with paromomycin resistance in conidia of heterokaryons hetero- 


\section{Table 2. Mitochondrial cytochrome ratios in strains carrying [cs-67] and suppressor mutations}

Cytochrome ratios \pm standard error of the mean were calculated as described in Methods from room temperature difference spectra (Fig. 2). Three to five independent measurements of the same mitochondrial sample were made. Only in the case of the wild-type, [cs-67], sumA35 [cs-67] and sumA35 sumB19 [cs-67] strains was more than one independent sample prepared.

\begin{tabular}{|c|c|c|c|c|c|c|}
\hline \multirow{3}{*}{$\begin{array}{c}\text { Strain } \\
\text { (relevant genotype) }\end{array}$} & \multicolumn{6}{|c|}{ Cytochrome ratios } \\
\hline & \multicolumn{3}{|c|}{ Grown at $37^{\circ} \mathrm{C}$} & \multicolumn{3}{|c|}{ Grown at $20^{\circ} \mathrm{C}$} \\
\hline & $c: a a_{3}$ & $c: b$ & $b: a a_{3}$ & $c: a a_{3}$ & $c: b$ & $b: a a_{3}$ \\
\hline Wild-type & $1 \cdot 30 \pm 0 \cdot 06$ & $0.95 \pm 0.03$ & $1.37 \pm 0.06$ & $1.61 \pm 0.09$ & $1.01 \pm 0.08$ & $1 \cdot 59 \pm 0 \cdot 18$ \\
\hline$[c s-67]$ & $1.35 \pm 0.09$ & $0.97 \pm 0.03$ & $1 \cdot 39 \pm 0 \cdot 10$ & $4 \cdot 65 \pm 0.23$ & $2 \cdot 14 \pm 0.16$ & $2 \cdot 15 \pm 0 \cdot 17$ \\
\hline$[c s-67$ sumD16] & $1 \cdot 40$ & 1.03 & 1.39 & $3.00 \pm 0.15$ & $1 \cdot 40 \pm 0 \cdot 11$ & $2 \cdot 14 \pm 0 \cdot 17$ \\
\hline sumA31 [cs-67] & $1.86 \pm 0.08$ & $1.08 \pm 0.02$ & $1 \cdot 78 \pm 0 \cdot 16$ & $3.23 \pm 0.21$ & $1.53 \pm 0.08$ & $2 \cdot 11 \pm 0.23$ \\
\hline $\operatorname{sum} A 35[\mathrm{cs}-67]$ & $2 \cdot 56 \pm 0 \cdot 19$ & $1 \cdot 13 \pm 0.03$ & $2 \cdot 24 \pm 0 \cdot 19$ & $2 \cdot 80 \pm 0 \cdot 18$ & $1.33 \pm 0.09$ & $2 \cdot 10 \pm 0 \cdot 15$ \\
\hline $\operatorname{sumB19}[\mathrm{cs}-67]$ & $3.09 \pm 0.03$ & $1.27 \pm 0.04$ & $2.44 \pm 0.06$ & $4 \cdot 01$ & 1.56 & $2 \cdot 61$ \\
\hline $\operatorname{sum} C 40[\mathrm{cs}-67]$ & $2.06 \pm 0.09$ & $1 \cdot 14 \pm 0.04$ & $1.80 \pm 0.07$ & $4 \cdot 05 \pm 0 \cdot 21$ & $1.75 \pm 0.06$ & $2 \cdot 31 \pm 0 \cdot 14$ \\
\hline sumE83 [cs-67] & $2.08 \pm 0.04$ & $1 \cdot 12 \pm 0.02$ & $1.86 \pm 0.03$ & $4.65 \pm 0.22$ & $2 \cdot 10 \pm 0 \cdot 20$ & $2 \cdot 21 \pm 0 \cdot 20$ \\
\hline $\operatorname{sumA31} \operatorname{sumB19}[\mathrm{cs}-67]$ & $3.50 \pm 0.05$ & $1 \cdot 52 \pm 0 \cdot 12$ & $2 \cdot 30 \pm 0 \cdot 15$ & $4 \cdot 42^{-}$ & 1.61 & $2 \cdot 73$ \\
\hline $\operatorname{sum} A 35 \operatorname{sum} B 19[\mathrm{cs}-67]$ & $3 \cdot 80 \pm 0 \cdot 21$ & $1.53 \pm 0.05$ & $2.48 \pm 0.08$ & $4 \cdot 62 \pm 0.25$ & $1 \cdot 73 \pm 0 \cdot 20$ & $2 \cdot 67 \pm 0 \cdot 16$ \\
\hline
\end{tabular}

zygous for the [cs-67] gene. This was only clearly seen on complete medium over a narrow concentration range $\left(0.4\right.$ to $\left.0.8 \mathrm{mg} \mathrm{ml}^{-1}\right)$. The conferment of resistance to paromomycin by [cs-67] was more striking in strains carrying sumA35, sumB19 and sumC40. This was because the mutations resulted per se in hypersensitivity to paromomycin, while sumA31, sumE83 and the mitochondrially inherited [sumD16] were indistinguishable from wild-type. [cs-67] was epistatic to all suppressor mutations vis-à-vis paromomycin resistance.

\section{Mitochondrial cytochrome spectra}

Spectra of mutants carrying [cs-67] and one or more nuclear suppressors (Fig. 2) showed that none of these mutants had normal cytochrome complements at either 37 or $20^{\circ} \mathrm{C}$. The spectra, which were obtained from samples of comparable protein concentration, and the ratios of cytochrome $c: a a_{3}$ (Table 2) suggested that many of the suppressors partially restored the low level of cytochrome $a a_{3}$ found in the cold-sensitive strain at $20^{\circ} \mathrm{C}$. Given this and the fact that the ratio of cytochrome $b: a a_{3}$ in each strain carrying [cs-67] and a suppressor, grown at $20^{\circ} \mathrm{C}$, was never significantly less than that of the cold-sensitive strain at $20^{\circ} \mathrm{C}$ (Table 2), it seemed that these suppressors were modifying the level of cytochrome $b$ at the same time as that of cytochrome $a a_{3}$. This was distinct from the situation found at $37^{\circ} \mathrm{C}$ where mutants carrying [cs-67] and a nuclear suppressor showed a deficiency in cytochrome $a a_{3}$ with respect to the cytochrome $c$ level, which varied with each suppressor, but a minimal deficiency or no alteration in cytochrome $b$ with respect to the cytochrome $c$ level.

The mitochondrially inherited suppressor [sumD16] partially suppressed the modifications in the cytochrome spectra of [cs-67] at $20^{\circ} \mathrm{C}$, while the spectrum of the double mutant [cs-67 sumD16] was indistinguishable from that of the wild-type and cold-sensitive strains at $37^{\circ} \mathrm{C}$ (Fig. 2 and Table 2).

At 20 and $37^{\circ} \mathrm{C}$ the growth rate of suppressor mutants on solid medium did not correlate with the degree to which the cytochrome spectra were modified. For example, at $37^{\circ} \mathrm{C}$, the mutant sumB19 [cs-67] had more abnormal cytochrome ratios than sumA35 [cs-67], despite the fact that the latter grew less well on solid complete medium.

\section{Thermolability of cytochrome oxidase}

As some of the strains carrying [cs-67] and two suppressors were partially heat-sensitive, the cytochrome oxidase activity of these strains should have been more heat-sensitive than 


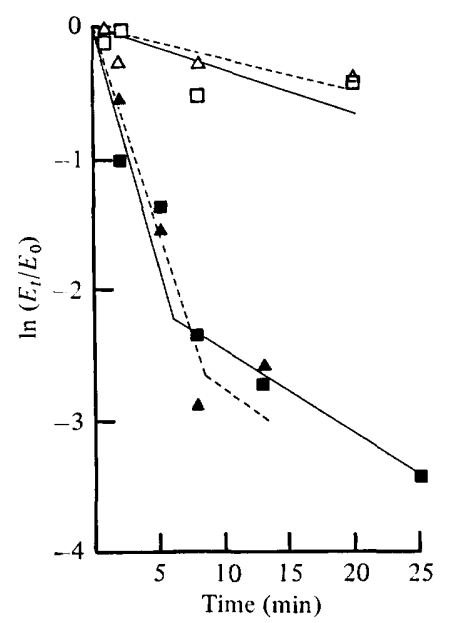

Fig. 3. Heat inactivation of cytochrome oxidase from the wild-type strain $(\square-\square)$ and mutant sumA35 sumB19 [cs-67] $(\triangle---\triangle)$. The enzyme activity was $E_{0}$ at time 0 and $E_{t}$ at time $t$, where $t$ was the duration of heat treatment. Open and filled symbols represent heat treatment at 40 and $60^{\circ} \mathrm{C}$, respectively.

Table 3. Assay of respiratory enzymes of wild-type, cold-sensitive and chloramphenicol-treated wild-type strains

Mycelium was grown at $20^{\circ} \mathrm{C}$, in the presence of chloramphenicol where indicated, and mitochondrial samples were prepared using a grind-mill (see Methods). Values are the means of assays performed on mitochondrial fractions banding on discontinuous sucrose gradients at the $1 \cdot 20 / 1 \cdot 35$ and $1 \cdot 35 / 1.60 \mathrm{M}$ interfaces.

$\begin{array}{ccc}\text { Strain } & \begin{array}{c}\text { Chloramphenicol } \\ \left(\mathrm{mg} \mathrm{ml}^{-1}\right)\end{array} & \begin{array}{c}\text { oxidase } \\ (\mathrm{nmol} \mathrm{O} \\ \left.\mathrm{min}^{-1} \mathrm{mg}^{-1}\right)\end{array}\end{array}$

Succinate-
cytochrome $c$
reductase
$\left(\right.$ nmol $\left.\min ^{-1} \mathrm{mg}^{-1}\right)$

$\begin{array}{lrrrr}\text { Wild-type } & - & 240 & 980 & 7 \cdot 6 \\ \text { [cs-67] } & - & 56 & 890 & 8 \cdot 7 \\ \text { Wild-type } & 2 \cdot 5 & 155 & 2530 & 10 \cdot 9 \\ \text { Wild-type } & 6 \cdot 0 & 84 & 955 & 8 \cdot 3\end{array}$

Succinate dehydrogenase $\left(\Delta A \mathrm{~min}^{-1} \mathrm{mg}^{-1}\right)$

that of the wild-type if [cs-67] and the suppressors directly modified cytochrome oxidase. However, the cytochrome oxidase activity of the mutant with the most extreme heatsensitive phenotype, sumA35 sumB19 [cs-67], was similar to that of the wild-type (Fig. 3) indicating that the mutations involved did not directly modify cytochrome oxidase subunits.

\section{Analysis of components of the respiratory chain}

The results in Table 3 show that the cold-sensitive strain grown at $20^{\circ} \mathrm{C}$ has a reduced cytochrome oxidase activity but normal succinate dehydrogenase and succinate-cytochrome $c$ reductase (this involves the cytochrome $b c_{1}$ complex - Complex III). As three subunits of cytochrome oxidase and one subunit of Complex III are synthesized in the mitochondria of yeast and N. crassa (Katan et al., 1976; Lin et al., 1978; Weiss, 1972), this suggested that [cs-67] directly affects cytochrome oxidase rather than mitochondrial protein synthesis. This conclusion became less tenable as a result of a control experiment in which it was shown that the succinate-cytochrome $c$ reductase activity of the wild-type strain was not decreased when grown in the presence of the mitoribosomal inhibitor chloramphenicol (Table 3).

Figure 4 shows that the cytochrome content of the cold-sensitive strain grown at $20^{\circ} \mathrm{C}$ was very similar to that of the wild-type grown in the presence of chloramphenicol. Both had a cytochrome $a a_{3}$ deficiency but the effect on cytochrome $b$ was not clear. 


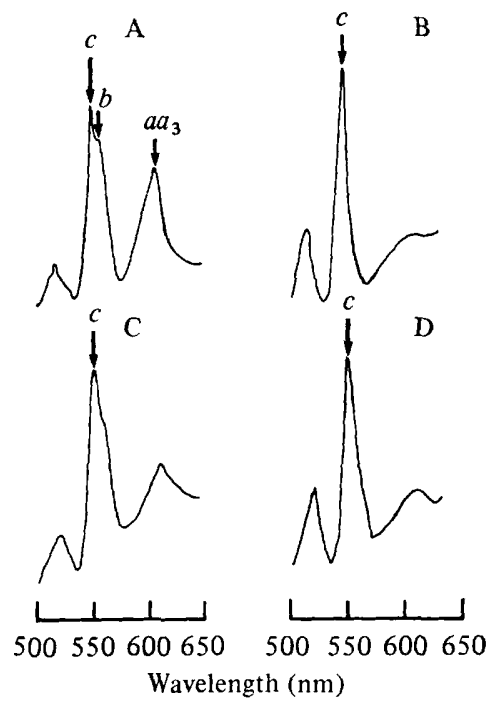

Fig. 4. Room temperature difference spectra: A, wild-type; B, [cs-67]; C and D, wild-type grown in the presence of 2.5 and $6.0 \mathrm{mg}$ chloramphenicol ml $\mathrm{m}^{-1}$, respectively. Mycelium was grown at $20^{\circ} \mathrm{C}$, and mitochondria were isolated using a grind-mill (see Methods). Spectral studies were performed on a fraction banding at the 1.20/1.35 $\mathrm{M}$ interface of a discontinuous sucrose gradient.

\section{Protein profiles of mitochondrial membrane preparations on gradient gels}

Membrane preparations were made from the wild-type, the cold-sensitive strain, a strain having the mitochondrial genotype [cs-67 sumD16] and the wild-type grown in the presence of 2.5 and $6.0 \mathrm{mg}$ chloramphenicol ml $\mathrm{ml}^{-1}$. (At $20^{\circ} \mathrm{C}$ in the presence of $6.0 \mathrm{mg}$ chloramphenicol $\mathrm{ml}^{-1}$, the wild-type grew at approximately the same rate as the cold-sensitive strain.)

SDS gradient acrylamide gels exposed a few reproducible differences in these preparations, for proteins of molecular weight between 60000 and 10000 (outside these limits, the gels were less reproducible). The bands on the gels have been numbered arbitrarily beginning with a band of apparent molecular weight 52000 . Under the experimental conditions used here, the following band differences were observed in the mitochondrial membrane preparations from the cold-sensitive strain with respect to those from the wild-type: a decrease in bands 5, 10a, 18 and 24 and an increase in bands 8, 9 and 23 (Fig. 5). The mitochondrial membrane preparations from the wild-type strain grown in the presence of chloramphenicol were virtually identical to those from the cold-sensitive strain grown at $20^{\circ} \mathrm{C}$ except for the following differences: a decrease in band 8 , a slight decrease in band 9 and a slight increase in band 23 (Fig. 5). It appeared that increasing the concentration of chloramphenicol in the growth medium from $2 \cdot 5$ to $6 \cdot 0 \mathrm{mg} \mathrm{ml}^{-1}$ only significantly affected band 8 .

Although a mitochondrial membrane sample was only made once from a strain carrying [cs-67 sum D16], the results (not shown) suggested that, with respect to the alterations caused by [cs-67], [sumD16] fully suppressed the increase in bands 9 and 23, partially suppressed the increase in band 8, partially suppressed the decrease in band 18 and had no effect on the decrease in bands 5 and 24 (band 10a was not resolved on a gel containing this preparation).

By calibrating the gels with standard proteins, as described in Methods, the following bands had the apparent molecular weights indicated: 5 (43000), 8 (35000), 9 (34000), 10a (33000), 18 (20000), 23 (15000), 24 (14000). 

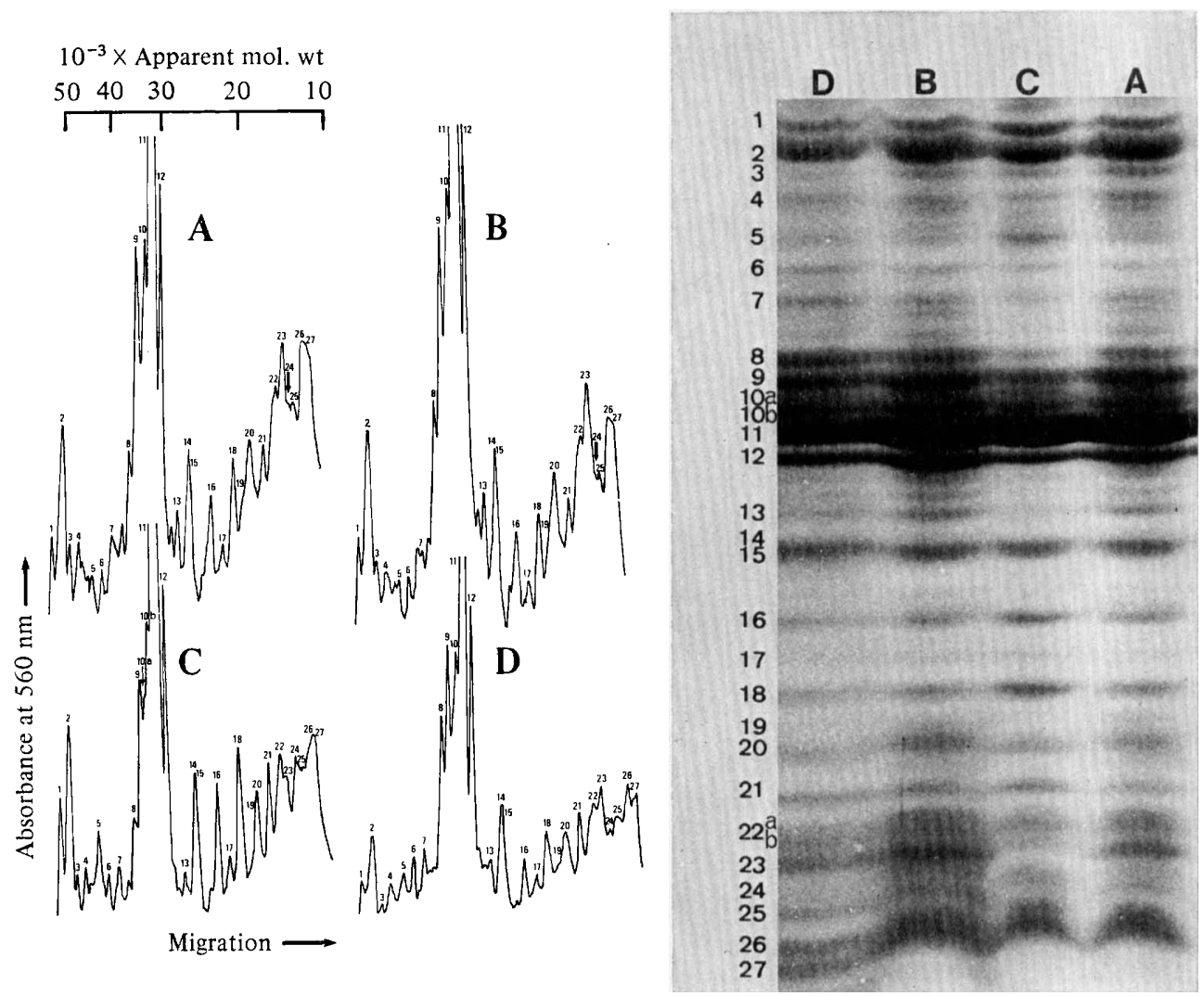

Fig. 5. Mitochondrial membrane protein profiles on SDS gradient acrylamide gels: A and B, wild-type grown in the presence of 2.5 and $6.0 \mathrm{mg}^{\circ}$ chloramphenicol ml ${ }^{-1}$, respectively; C, wild-type; $\mathrm{D}$, mutant [cs-67]. Mycelium was grown at $20^{\circ} \mathrm{C}$ and membranes were prepared from the mitochondrial fractions which banded at the 1.20/1.35 $\mathrm{M}$ interface of a discontinuous sucrose gradient. The scans are of the tracks shown in the photograph.

\section{DISCUSSION}

Suppressors of the mitochondrially inherited mutation [cs-67] mapped in four nuclear genes and one mitochondrial gene. The most obvious hypothesis to account for this pattern was that the [cs-67] gene and five suppressor genes participated in specifying the assembly of a mitochondrial component. However, it should be stated clearly that there was no evidence that any of the genes participated directly in coding for a subunit of the component, rather than in a number of possible indirect ways. Indeed, as only the [cs-67] allele was available we could not exclude rigorously the seemingly far-fetched hypothesis that one or more of the suppressors were translational suppressors.

The spectral properties of [cs-67] suggested cytochrome oxidase as the possible primary product of the [cs-67] gene. The results presented here made this hypothesis unlikely. The triple mutant sumA35 sumB19 [cs-67] resulting in a heat-sensitive phenotype did not result in a heat-sensitive cytochrome oxidase. This did not exclude that [cs-67] might be involved specifically, but indirectly, in the synthesis or assembly of cytochrome oxidase, in a posttranslational or regulatory capacity or perhaps, as in the box mutations in $S$. cerevisiae, in the regulation of both cytochrome oxidase and cytochrome $b$ (for review, see Borst \& Grivell, 1978). However, the lack of correlation of growth rate with cytochrome content suggested that in [cs-67] strains cytochrome deficiencies were not primarily the factor limiting growth. 
Two lines of evidence pointed to the mitoribosome as the primary target of the [cs-67] mutation. Firstly, the alterations in the pattern of mitochondrial membrane protein by the [cs-67] mutation mimicked those produced by sub-lethal concentrations of chloramphenicol, an inhibitor of mitochondrial protein synthesis in yeast (Davey et al., 1969). N. crassa (Kuntzel, 1969) and, almost undoubtedly, A. nidulans (Lazarus \& Turner, 1977). Some caution, however, should be exercised before giving this analogy too much weight. Growth in the presence of chloramphenicol did not seem to decrease the activity of succinatecytochrome $c$ reductase (a possible reason for this may be found in the work of Beattie et al., 1974). Chloramphenicol inhibition of the synthesis of cytochrome $b$ was ambiguous and the effect on the protein profile of the mitochondrial membrane was limited. It must be stressed that in the chloramphenicol treatment, total protein rather than de novo synthesized protein was detected in the gels. Only experiments following the specific incorporation of label into the chloramphenicol sensitive bands can clarify this point.

The second line of evidence concerned the action of paromomycin. This antibiotic inhibits protein synthesis at the level of the ribosome in E. coli (Weisblum \& Davies, 1968) and causes mistranslation in an in vitro system employing the cytoplasmic ribosomes of Tetrahymena (Palmer \& Wilhelm, 1978). In S. cerevisiae, paromomycin inhibits mitochondrial protein synthesis (Davey et al., 1970) and mutations resistant to paromomycin map in the mitochondrial genome. The suppressor mutations, while suppressing the cold-sensitivity, did not suppress the paromomycin resistance of [cs-67]. Incidentally, this argued against the sum mutations representing translational rather than physiological suppressors.

It should be mentioned that, while the evidence pointed to the mitoribosome as the primary target of the [cs-67] mutation, other interpretations were still possible, for example, [cs-67] could have been affecting a component of the mitochondrial membrane, resulting in both defective integration of cytochrome oxidase and cytochrome $b$ at $20^{\circ} \mathrm{C}$ and decreased permeability to paromomycin at all temperatures.

Mutations in the mitochondrially inherited suppressor [sumD] recombined with [cs-67] at a frequency sufficient to suggest that these two phenotypes represented mutations in different genes. However, the recent data pointing to the presence of introns one or more kilobases long in mitochondrial genes of yeast (for review, see Borst \& Grivell, 1978) calls for caution in interpreting recombination data.

The results presented in this paper constitute the genetic characterization of a system of nuclear-mitochondrial interactions. Further clarification of the nature of the system can only be achieved by biochemical studies of the components of the mitochondrial membrane and the mitoribosome.

We would like to thank Dr H. N. Arst, Jr for critical reading of the manuscript. During this work, R. B. Waring was in receipt of a Science Research Council Studentship.

\section{REFERENCES}

Alderson, T. \& Hartley, M. J. (1969). Specificity for spontaneous and induced mutations at several gene loci in Aspergillus nidulans Mutation Research 8, 255-264.

Arst, H. N., JR \& Cove, D. J. (1969). Methylammonium resistance in Aspergillus nidulans. Journal of Bacteriology 98, 1284-1293.

Beattie, D. S., Lin, L.-F. H. \& Stuchell, R. N. (1974). Studies on the control of mitochondrial protein synthesis in yeast. In The Biogenesis of Mitochondria, pp. 465-475. Edited by A. M. Kroone \& C. Saccone. New York: Academic Press.

Bertrand, H. \& Collins, R. A. (1978). A regulatory system controlling the production of cyto- chrome $a a_{3}$ in Neurospora crassa. Molecular and General Genetics 166, 1-13.

Bolotin-Fukuhara, M., Faye, G. \& Fukuhara, H. (1977). Temperature sensitive respiratorydeficient mitochondrial mutations: isolation and genetic mapping. Molecular and General Genetics 152, 295-305.

Borst, P. \& Grivell, L. A. (1978). The mitochondrial genome of yeast. Cell 15, 705-723.

Briggs, M. M. \& Capaldi, R. A. (1977). Near neighbour relationships of the subunits of cytochrome $c$ oxidase. Biochemistry 16, 73-77.

Butcher, R. G. (1970). Studies on succinate oxidation. I. The use of intact tissue sections. Experimental Cell Research 60, 54-60. 
Chance, B. \& Williams, G. R. (1955). Respiratory enzymes in oxidative phosphorylation. II. Difference spectra. Journal of Biological Chemistry 217, 395-407.

Clutterbuck, A. J. \& Cove, D. J. (1974). Linkage map of Aspergillus nidulans. In CRC Handbook of Microbiology, vol. IV, pp. 665-676. Edited by A. I. Laskin \& H. A. Lechevalier. Cleveland, Ohio: Chemical Rubber Co.

COVE, D. J. (1966). The induction and repression of nitrate reductase in the fungus Aspergillus nidulans. Biochimica et biophysica acta 113, 51-56.

Davey, P. J., YU, R. \& Linnane, A. W. (1969). The intracellular site of formation of the mitochondrial protein synthetic system. Biochemical and Biophysical Research Communications 36, 30-34.

Davey, P. J., Haslam, J. M. \& Linnane, A. W. (1970). Biogenesis of mitochondria. 12. The effects of aminoglycoside antibiotics on the mitochondrial and cytoplasmic protein synthesising systems of $S$. cerevisiae. Archives of Biochemistry cind Biophysics 136, 54-64.

Devenish, R. J., English, K. J., Hall, R. M., LinNane, A. W. \& Lukins, H. B. (1978). Biogenesis of mitochondria. 49. Identification and mapping of a new mitochondrial locus (tsr 1 ) which maps within the polar region of the yeast mitochondrial genome. Molecular and General Genetics 161, 251-259.

Dunker, A. K. \& Rueckert, R. R. (1969). Observations on molecular weight determinations on polyacrylamide gels. Journal of Biological Chemistry 244, 5074-5080.

Ferguson-Miller, S. S., Brautigan, D. L. \& Margoliash, E. (1976). Correlation of the kinetics of electron transfer activity of various eukaryotic cytochromes $c$ with binding to mitochondrial cytochrome $c$ oxidase. Journal of Biological Chemistry 251, 1104-1115.

Goffeau, A., Labaille, F., Mohar, O. \& Colson, A.-M. (1976). Screening tests for suppressors of respiratory-deficient mutants in Schizosaccharomyces pombe and model for a mitochondrial partial suppression of a nuclear pleiotropic strain. In Genetics and Biogenesis of Chloroplasts and Mitochondria, pp. 851-856. Edited by T. Bucher, W. Neupert, W. Sebald \& S. Werner. Amsterdam: North Holland Biomedical Press.

GoRINI, L. (1974). Streptomycin and misreading of the genetic code. In Ribosomes, pp. 791-803. Edited by M. Nomura, A. Tissieres \& P. Lengyel. Cold Spring Harbor, N.Y.: Cold Spring Harbor Laboratory.

Grivell, L. A., Netter, P., Borst, P. \& Slonimski, P. P. (1973). Mitochondrial antibiotic resistance in yeast: ribosomal mutants resistant to chloramphenicol, erythromycin and spiramycin. Biochimica et biophysica acta 312, 358-367.

GunAtilleke, I. A. U. N. (1974). Biochemistry and genetics of mitochondrial function in Aspergillus nidulans. Ph.D. thesis, University of Cambridge.

Gunatilleke, I. A. U. N., ARST, H. N., JR \& Scazzocchio, C. (1975a). Three genes determine the carboxin sensitivity of mitochondrial succinate oxidation in Aspergillus nidulans. Genetical Research 26, 297-305.

Gunatilleke, I. A. U. N., Scazzocchio, C. \&
ARsT, H. N., JR (1975b). Cytoplasmic and nuclear mutations to chloramphenicol resistance in Aspergillus nidulans. Molecular and General Genetics 137, 269-276.

Henry, M.-F. \& Nyns, E. J. (1975). Cyanideinsensitive respiration. An alternative mitochondrial pathway. Sub-cellular Biochemistry 4, 165.

InOUE, Y., IshizUKA, K. \& Mitsui, S. (1967). Inhibition of respiration of yeast by photosynthesis inhibiting herbicides. Agricultural and Biological Chemistry 31, 422-427.

Katan, M. B., Harten-Loosbroek, N. \& Groot, G. S. P. (1976). The cytochrome $b c_{1}$ complex of yeast mitochondria. Site of translation of the polypeptides in vivo. European Journcl of Biochemistry 70, 409-417.

KING, T. E. (1967). Preparations of succinatecytochrome $c$ reductase and the cytochrome $b c_{1}$ particle reconstitution of succinate-cytochrome $c$ reductase. Methods in Enzymology 10, 216-225.

Kirschmann, C. \& Davis, B. D. (1969). Phenotypic suppression in Escherichia coli by chloramphenicol and other reversible inhibitors of the ribosome. Journal of Bacteriology 98, 152-159.

Kohout, J. \& Bertrand, H. (1976). Nuclear suppressors of the poky cytoplasmic mutant in Neurospora crassa. I. Genetic and respiratory properties. Canadian Journal of Genetics and Cytology 18, 311-324.

KunTZel, H. (1969). Specificity of mitochondrial and cytoplasmic ribosomes from Neurospora crassa in poly(U)-dependent cell-free systems. FEBS Letters 4, 140-142.

LAEMMLI, U. K. (1970). Cleavage of structural proteins during the assembly of the head of bacteriophage T4. Nature, London 227, 680-685.

Lambin, P., Rochu, D. \& Fine, J. M. (1976). A new method for determination of molecular weights of proteins by electrophoresis across a sodium dodecyl sulphate (SDS) polyacrylamide gradient gel. Analytical Biochemistry 74, 567-575.

Lambowitz, A. M., Slayman, C. W., Slayman, C. L. \& BonNER, W. D., JR (1972a). The electron transport components of wild-type and poky strains of Neurospora crassa. Journal of Biological Chemistry 247, 1536-1545.

Lambowitz, A. M., Smith, E. W. \& Slayman, C. W. $(1972 b)$. Electron transport in Neurospora mitochondria. Studies on wild-type and poky. Journal of Biological Chemistry 247, 4850-4858.

LARDY, H. A., Johnson, D. \& McMurray, W. C. (1958). Antibiotics as tools for metabolic studies. I. A survey of toxic antibiotics in respiratory, phosphorylative and glycolytic systems. Archives of Biochemistry and Biophysics 78, 587-597.

LAZZARUS, C. M. \& TURNER, G. (1977). Extranuclear recombination in Aspergillus nidulans: closely linked multiple chloramphenicol and oligomycin resistance loci. Molecular and General Genetics 156, 303-311.

Lin, L. H., Clejan, L. \& Beattie, D. S. (1978). The synthesis of cytochrome $b$ on mitochondrial ribosomes in baker's yeast. European Journal of Biochemistry 87, 171-179.

Mackintosh, M. E. \& Pritchard, R. H. (1963). The production and replica plating of micro- 
colonies of Aspergillus nidulans. Genetical Research 4, 320-322.

McCully, K. S. \& Forbes, E. (1965). The use of p-fluorophenylalanine with 'master strains' of Aspergillus nidulans for assigning genes to linkage groups. Genetical Research 6, 352-359.

Mitchell, M. B. \& Mitchell, H. K. (1956). A nuclear gene suppressor of a cytoplasmically inherited character in Neurospora crassa. Journal of General Microbiology 14, 84-89.

Nomura, M., Morgan, E. A. \& Jaskunas, S. R. (1977). Genetics of bacterial ribosomes. Annual Review of Genetics 11, 297-347.

Palmer, E. \& Wilhelm, J. M. (1978). Mistranslation in a eukaryotic organism. Cell 13, 329-354.

Pontecorvo, G., Roper, J. A., Hemmons, L. M., Macdonald, K. D. \& Bufton, A. W. J. (1953). The genetics of Aspergillus nidulans. Advances in Genetics 5, 141-238.

REVER, B. M. (1965). Biochemical and genetical studies of inorganic nitrogen metabolism in Aspergillus nidulans. Ph.D. thesis, University of Cambridge.

Rowlands, R. T. \& Turner, G. (1973). Nuclear and extranuclear inheritance of oligomycin resistance in Aspergillus nidulans. Molecular and General Genetics 126, 201-216.

Rowlands, R. T. \& Turner, G. (1974a). Physiological and biochemical studies of nuclear and extranuclear oligomycin-resistant mutants of Aspergillus nidulans. Molecular and General Genetics 132, 73-88.

Rowlands, R. T. \& TuRner, G. (1974b). Recombination between the extranuclear genes conferring oligomycin resistance and cold-sensitivity in Aspergillus nidulans. Molecular and General Genetics 133, 151-161.

Rowlands, R. T. \& TuRner, G. (1975). Threemarker extranuclear mitochondrial crosses in Aspergillus nidulans. Molecular and Genercil Genetics 141, 69-79.

SaINSARd, A. (1975). Mitochondrial suppressor of a nuclear gene in Paramecium. Nature, London 257 , 312-314.

Schatz, G. \& Mason, T. L. (1974). The biosynthesis of mitochondrial proteins. Annual Review of Biochemistry 43, 51-87.

Singh, A., Mason, T. L. \& ZimmermanN, R. A. (1978). A cold-sensitive cytoplasmic mutation of Saccharomyces cerevisiae affecting assembly of the mitochondrial 50 S ribosomal subunit. Molecular and General Genetics 161, 143-151.

Spithill, T., English, K. J. \& NaGley, P. (1978). Altered mitochondrial ribosomes in a coldsensitive mutant of Saccharomyces cerevisiae. Molecular Biology Reports 4, 83-86.
Storm, E. M. \& Marmur, J. (1975). A temperature sensitive mitochondrial mutation of Saccharomyces cerevisiae. Biochemical and Biophysical Research Communications 64, 752-759.

Trembath, M. K., Monk, B. C., Kellerman, G. M. \& Linnane, A. W. (1975a). Biogenesis of mitochondria. 50. Phenotypic suppression of a mitochondrial mutation by a nuclear gene in Saccharomyces cerevisiae. Molecular and General Genetics 140, 333-337.

Trembath, M. K., Monk, B. C., Kellerman, G. M. \& Linnane, A. W. (1975b). Biogenesis of mitochondria. 36. The genetic and biochemical analysis of a mitochondrially determined coldsensitive oligomycin resistant mutant of Saccharomyces cerevisiae with affected mitochondrial ATPase assembly. Molecular and General Genetics 141, 9-22.

Turner, G. \& Rowlands, R. T. (1976). Cytochrome abnormalities and cyanide-resistant respiration in extranuclear mutants of Aspergillus nidulans. Journal of Bacteriology 125, 389-397.

Turner, G. \& Rowlands, R. T. (1977). Mitochondrial genetics of Aspergillus nidulans. In Genetics and Physiology of Aspergillus, pp. 319337. Edited by J. E. Smith \& J. A. Pateman. London \& New York: Academic Press.

Waldron, C. \& Roberts, C. F. (1973). Cytoplasmic inheritance of a cold-sensitive mutant in Aspergillus nidulans. Journal of Generel Microbiology $\mathbf{7 8}$, 379-381.

WARDEN, J. T. \& EdWARDS, D. L. (1976). Electron spin resonance investigations of mitochondrial electron transport in Neurospora crassa. European Journcl of Biochemistry 71, 411-418.

Weisblum, B. \& Davies, J. (1968). Antibiotic inhibitors of the bacterial ribosome. Bacteriological Reviews 32, 493-528.

WeISS, H. (1972). Cytochrome $b$ in Neurospora crassa mitochondria. A membrane protein containing subunits of cytoplasmic and mitochondrial origin. European Journal of Biochemistry 30, 469-478.

Weiss, H., von Jagow, G. \& KlingenberG, M. (1970). Characterisation of Neurospora crassa mitochondria prepared with a grind-mill. European Journal of Biochemistry 14, 75-82.

Wharton, D. C. \& Tzagoloff, A. (1967). Cytochrome oxidase from beef heart mitochondria. Methods in Enzymology 10, 245-250.

YoNETANI, T. (1960). Studies on cytochrome oxidase. I. Absolute and difference absorption spectra. Journal of Biological Chemistry 235, 845-852. 